\title{
Exercise-induced bronchospasm: implications for patients with or without asthma in primary care practice
}

This article was published in the following Dove Press journal:

International Journal of General Medicine

17 November 201।

Number of times this article has been viewed

\author{
Stuart W Stoloff' \\ Gene L Colice ${ }^{2}$ \\ Mary Lou Hayden ${ }^{3}$ \\ Timothy J Craig ${ }^{4}$ \\ Nancy K Ostrom ${ }^{5}$ \\ Nemr S Eid ${ }^{6}$ \\ Jonathan P Parsons ${ }^{7}$ \\ 'University of Nevada School of \\ Medicine, Reno, NV, ${ }^{2}$ Washington \\ Hospital Center, Washington, DC, \\ ${ }^{3}$ University of Virginia, Charlottesville, \\ VA, ${ }^{4}$ Pennsylvania State University, \\ Hershey, PA, ${ }^{5}$ Allergy and Asthma \\ Medical Group and Research Center, \\ San Diego, CA, ${ }^{6}$ University of \\ Louisville, Louisville, KY, ${ }^{7}$ Ohio State \\ University Asthma Center, Columbus, \\ $\mathrm{OH}, \mathrm{USA}$
}

\begin{abstract}
Exercise-induced bronchospasm (EIB) can represent a substantial barrier to physical activity. We present the cases of two patients with EIB, one with asthma, and one without asthma, who were evaluated at our primary care practice. The first case was a 44-year-old man with a history of seasonal allergic rhinitis but no asthma, who reported difficulty breathing when playing tennis. The second case was a 45 -year-old woman who presented with persistent, generally well-controlled asthma, who was now experiencing bouts of coughing and wheezing during exercise. In both cases, an exercise challenge was used to diagnose EIB, and patients were prescribed a short-acting beta agonist to be used immediately before initiating exercise. EIB is a frequently encountered problem among patients presenting to primary care specialists. Affected patients should be made aware of the importance of proactive treatment with a shortacting beta agonist before initiating any exercise.
\end{abstract}

Keywords: asthma, compliance, exercise-induced bronchospasm

\section{Introduction}

All patients should be encouraged to maintain an appropriate exercise program. In adults aged 18-65 years with no underlying chronic disease, such an exercise program should consist of 30 minutes of moderate-intensity physical activity at least 5 days per week or vigorous-intensity exercise at least 3 days per week (www.exerciseismedicine.org). Given the recent increase in focus on preventive care, and the importance of physical activity for general health and wellness, exercise-induced bronchospasm (EIB) may represent a substantial barrier to physical activity in affected patients.

Although up to $90 \%$ of patients with asthma have been reported to have EIB, as many as $10 \%$ of patients who have no overt respiratory dysfunction may develop EIB. For patients with asthma, exercise-induced asthma has also been used to describe this condition, although many physicians believe this term is a misnomer because it seems to imply that exercise causes rather than exacerbates or triggers an attack of asthma. EIB is defined as a $15 \%$ decrease in peak expiratory flow or forced expiratory volume in 1 second $\left(\mathrm{FEV}_{1}\right)$ after physical exertion that increases baseline heart rate to $80 \%$ of maximum for 4-6 minutes, ${ }^{1}$ compared with pre-exercise baseline. This condition usually lasts 15-20 minutes after starting or shortly after completing moderately vigorous exercise. ${ }^{2,3}$ The mechanism underlying this effect is not fully understood, but the fundamental trigger is thought to involve drying and cooling of airways during exercise. This can cause sensitized mast cells to release pro-inflammatory mediators, which ultimately may cause bronchospasm. ${ }^{2}$
Correspondence: Stuart Stoloff Clinical Professor, Department of Family and Community Medicine, University of Nevada School of Medicine, $1664 \mathrm{~N}$. Virginia Street, Reno, NV 89557, USA

Tel +I 7758836888

Fax +I 7758836524

Email drstoloff@sbcglobal.net 
The fairly broad incidence of EIB strongly suggests that the majority of physicians in primary care practice will regularly encounter patients with EIB. Indeed, this condition is recognized as having appropriate management options by most primary care specialists. Despite the apparently uncomplicated nature of EIB as perceived by physicians, however, many patients are uncertain about how to manage this condition effectively. This may reflect their lack of awareness regarding existing treatment guidelines and the effective use of inhaled short-acting beta agonist (SABA) bronchodilators to mitigate exercise-induced respiratory symptoms due to bronchospasm. ${ }^{1}$ We present the cases of two patients with EIB, one with asthma, and one without asthma, who were recently evaluated at our primary care practice.

\section{Case presentation Case I}

A 44-year-old male with a history of seasonal allergic rhinitis, no smoking history, and no diagnosis of asthma presented to his primary care specialist reporting chest tightness, difficulty breathing, and wheezing when playing tennis. These respiratory symptoms only occurred with moderate-tovigorous activity. The symptoms were moderate but seemed to resolve with rest. The patient had a very active lifestyle, with a body mass index (BMI) of 25 but was experiencing increased shortness of breath when running on the tennis court once or twice a week. During intense exercise he would experience exercise-related dyspnea. Routine physical exam and diagnostic testing ruled out cardiovascular disease. Pulmonary function tests were ordered and revealed normal flow volume loop, normal lung volumes, and $\mathrm{FEV}_{1}$ that was $95 \%$ of predicted. The physician further requested a pulmonary exercise study using a treadmill, which showed a $>15 \%$ decline in $\mathrm{FEV}_{1}$ within 5 minutes after completing the exercise (Figure 1). Based on both medical history and exercise study results, the patient was provided with a SABA inhaler and instructed to use it at least 5 minutes before starting to exercise. The physician discussed with the patient that a warm-up period before exercise may reduce the degree of EIB and that a mask or scarf over the mouth may attenuate cold air-induced EIB. On follow-up, the patient no longer reported respiratory-related symptoms after exercise when he remembered to take his SABA prior to exercise.

\section{Case 2}

A 45-year-old woman presented to her primary care specialist with persistent asthma, which had been well controlled on low-to-medium dose-inhaled corticosteroid (ICS).

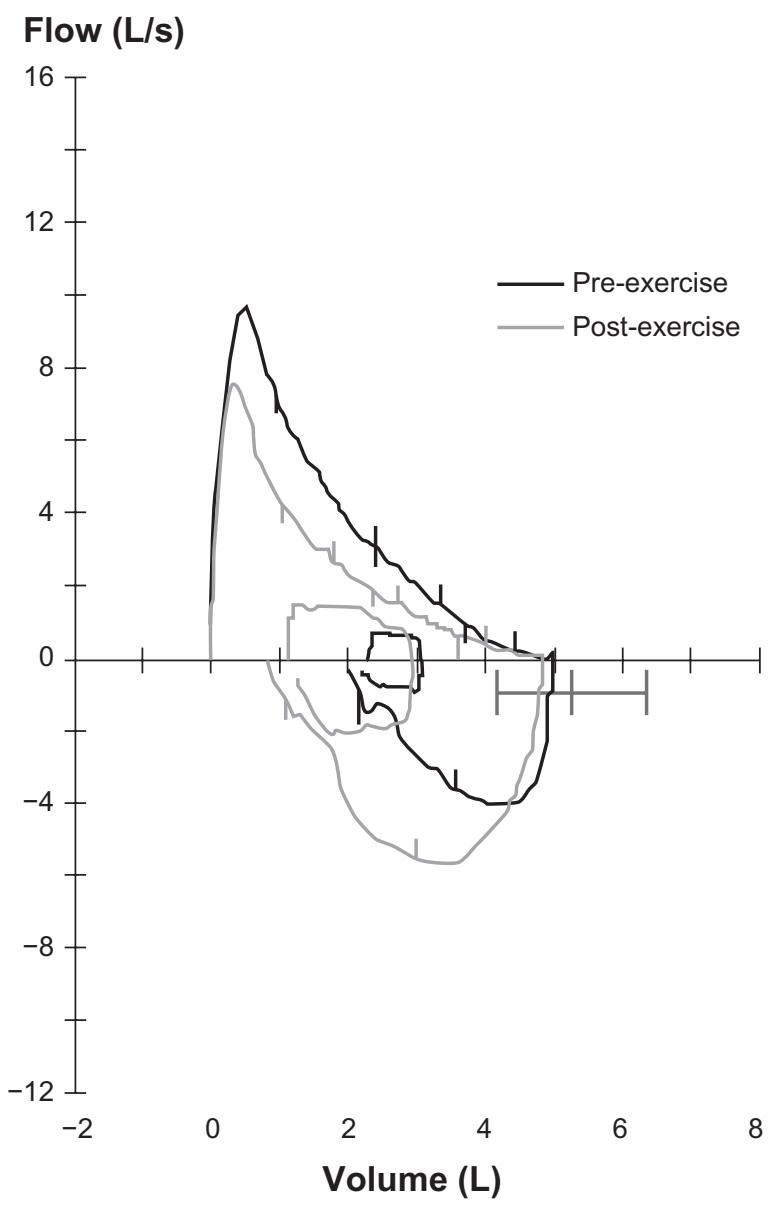

Figure I Spirometry results: pre-exercise challenge and post-exercise challenge.

The physician confirmed that the patient was taking her ICS as directed and that she had access to a rescue medication (albuterol). She had recently joined a gym where she participated in a strenuous aerobic exercise program two to three times a week; however, during each exercise session she experienced repeated bouts of coughing and wheezing, which forced her to take a break from the exercise. She denied experiencing shortness of breath, cough, or wheezing at night or at any other time except when exercising. Her baseline pulmonary function testing at rest was normal, and she had no history of emergency room visits, hospitalizations, or exacerbations of asthma requiring oral corticosteroids. The patient was frustrated with her inability to exercise vigorously and did not feel integrated with her peer group at the gym. She was not motivated to attend the most recent aerobic session because of her concern that she would not be able to keep up with the group exercise activity. Her primary care specialist ordered a pulmonary exercise study using a treadmill, which showed a $20 \%$ decline in $\mathrm{FEV}_{1}$ measured when cough and shortness of breath occurred during the exercise. The physician diagnosed EIB and educated the 
patient on the importance of taking her prescribed rescue medication prior to exercise; two puffs to be taken at least 5 minutes before each aerobic session to prevent the onset of her exercise-induced breathing difficulties. At follow-up 1 month later, the physician confirmed that the patient was taking her prescribed medications as directed for her asthma, that she was taking her albuterol prior to exercise, and that there were no other signs that she was experiencing asthma symptoms daily. The patient reported that she could participate fully in the aerobics session with no further episodes of breathing difficulty and did not experience any asthma symptoms with normal activities.

\section{Discussion}

Results from the recent EIB Landmark Survey (www. eiblandmarksurvey.com $)^{4}$ highlight the broad impact of EIB on patients' lives, including those without underlying asthma. The survey identifies exercise as the most common trigger for asthma symptoms, with almost one-third of all asthma patients reporting that exercise can trigger or exacerbate an asthma attack. Nearly half of respondents (46\%) report that their health interferes with their ability to participate in sports or recreation. As part of the EIB Landmark Survey, a general population cross-sectional survey was also done, in which more than one-quarter of all adult participants reported respiratory symptoms during or after physical activity, although less than $20 \%$ had actually been diagnosed with exerciseinduced asthma or EIB. The EIB Landmark Survey also confirmed that patients' understanding of exercise-related bronchospasm and its management differed from that of their health care providers. This is highlighted by the fact that only $41 \%$ of patients with exercise-related symptoms said that they took quick-relief medication at least sometimes before exercising, even though the standard treatment recommendation is to use a SABA medication at least 5 minutes before exercising. This lack of appropriate, preventative treatment in many patients may reflect misunderstanding between physicians and patients regarding proper management of EIB.

The significant manner in which asthma and EIB can affect quality of life is highlighted in the cases described in this report. Patients with EIB are less likely to engage in recreational activity and are, therefore, at risk of having increased BMI and its potential negative health sequelae. Furthermore, patients with seasonal allergic rhinitis, such as the example in Case 1, may be predisposed to EIB, with an estimated $40 \%$ of patients with allergic rhinitis also showing symptoms of EIB. ${ }^{5}$ In patients with underlying asthma, participation in physical activity without experiencing asthma symptoms is an important element of both optimal asthma management and quality of life. Therefore, EIB should be recognized and treated proactively to minimize barriers to participation in vigorous activities.

Guidelines for the Diagnosis and Management of Asthma indicate that patients with EIB should be monitored regularly to ensure that they have no symptoms of asthma in the absence of exercise. ${ }^{1}$ An exercise challenge test is often used for diagnosis because use of medical history alone lacks diagnostic accuracy and may result in false-positive or falsenegative diagnoses. Formal bronchoprovocation testing using eucapnic voluntary hyperventilation is the preferred method for diagnosis. In patients diagnosed with EIB, prophylactic treatment with a SABA bronchodilator before starting exercise is recommended and is reported to control symptoms in $80 \%-95 \%$ of asthma patients. ${ }^{1,5}$ Prophylactic bronchodilator medication may also be necessary in asthma patients who are stabilized on ICS maintenance therapy. As highlighted in Case 2, patients with mild, persistent asthma and concurrent EIB may require both an ICS to maintain adequate maintenance control and a SABA before exercise so that they do not develop asthma symptoms. In patients with chronic asthma, EIB can indicate poor underlying disease control ${ }^{1,6}$ and, hence, such patients should receive a global assessment of asthma management to ensure that they are taking their current medications as indicated. Furthermore, patients with EIB should also be educated on nonpharmacologic interventions that can be used to decrease the likelihood of an EIB episode. A warm-up period before exercise may reduce the degree of EIB, and a mask or scarf over the mouth may attenuate cold air-induced EIB. ${ }^{1,6}$ Finally, follow-up care is crucial, with re-evaluation of inhalation technique, the potential need for additional medication, availability of rescue medication, and ultimately, reassessment of the diagnosis in those patients who continue to show exercise-related symptoms. ${ }^{1,6}$

\section{Conclusion}

EIB is frequently encountered among patients presenting to primary care specialists. Effective management strategies are well established and can allow healthy levels of activity for improving quality of life. Primary care specialists should ensure that patients with EIB are aware of the importance of preventative treatment with a SABA before initiating exercise.

\section{Acknowledgments}

The EIB Landmark Survey was conducted by the national public opinion research organization Abt SRBI Inc, in 
partnership with Strategic Pharma Solutions, LLC, and was sponsored by Teva Respiratory, LLC. The authors wish to acknowledge the technical and editorial support provided by ApotheCom.

\section{Disclosure}

Dr Stoloff: Teva (grant/research support, consultant). Dr Colice: Teva (consultant, speakers' bureau), Boehringer Ingelheim (consultant, speakers' bureau), Pfizer (consultant, speakers' bureau), GlaxoSmithKline (consultant, speakers' bureau), Merck (consultant, speakers' bureau), Vatera (consultant, speakers' bureau), MedImmune (consultant, speakers' bureau). Ms Hayden: Teva (consultant), Dey Laboratory, Sunovian, Merck (speakers' bureau). Dr Craig: Teva (speakers' bureau). Dr Ostrom: Alcon (grant/research support), Amgen (grant/research support), Apotex (grant/research support), AstraZeneca (grant/research support, consultant, speakers' bureau), Boehringer Ingelheim (grant/research support), CRN (consultant), GlaxoSmithKline (grant/research support, speakers' bureau), HRA (grant/research support), ISTA (consultant), MedImmune (grant/research support),
Novartis (grant/research support), Proctor \& Gamble (grant/research support), Schering-Plough (grant/research support), Sunovion (Sepracor) (grant/research support), Teva (grant/research support, consultant, speakers' bureau). Dr Eid: Teva (consultant, speakers' bureau). Dr Parsons: Teva (consultant).

\section{References}

1. National Heart, Lung, and Blood Institute. Expert Panel Report 3 (EPR3): Guidelines for the Diagnosis and Management of Asthma. Bethesda, MD: National Institutes of Health, NIH Publication No. 07-4051, 2007. Available at: http://www.nhlbi.nih.gov/guidelines/asthma/asthgdln.htm. Accessed October 10, 2010.

2. Gotshall RW. Exercise-induced bronchoconstriction. Drugs. 2002; 62(12):1725-1739.

3. McFadden ER Jr, Gilbert IA. Exercise-induced asthma. $N$ Engl J Med. 1994;330(19):1362-1367.

4. EIB: A Landmark Survey [homepage]. Available at: www.eiblandmarksurvey.com. Accessed on October 20, 2011.

5. Milgrom H, Taussig LM. Keeping children with exercise-induced asthma active. Pediatrics. 1999;104(3):e38.

6. Weiler JM, Anderson SD, Randolph C, et al; American Academy of Allergy, Asthma and Immunology; American College of Allergy, Asthma and Immunology; Joint Council of Allergy, Asthma and Immunology. Pathogenesis, prevalence, diagnosis, and management of exerciseinduced bronchoconstriction: a practice parameter. Ann Allergy Asthma Immunol. 2010;105:S1-S47.

\section{Publish your work in this journal}

The International Journal of General Medicine is an international, peer-reviewed open-access journal that focuses on general and internal medicine, pathogenesis, epidemiology, diagnosis, monitoring and treatment protocols. The journal is characterized by the rapid reporting of reviews, original research and clinical studies across all disease areas.
A key focus is the elucidation of disease processes and management protocols resulting in improved outcomes for the patient.The manuscript management system is completely online and includes a very quick and fair peer-review system. Visit http://www.dovepress.com/ testimonials.php to read real quotes from published authors. 